\title{
A really serious conflict
}

\author{
Not all financial interests in drug discovery are detrimental, and many are essential for its success. But focusing on \\ perceived conflicts of interest may cause true scientific corruption to go unnoticed.
}

$\mathbf{T}^{\mathrm{T}}$ ransforming a laboratory finding into a new medicine involves numerous steps and stakeholders. In a simple case, a researcher discovers and publishes a new target, and an academic or industrial organization decides to commercialize it. After an initial period of development, this organization licenses the target or a lead compound to a pharmaceutical company with a view to take it to the market. The company sponsors clinical trials, the results of which are also published and evaluated by regulatory agencies that ultimately approve the new therapy for its use in humans. The company then promotes its new product to claim the largest possible share of the market. In an ideal scenario, a postmarketing follow-up of the compound gives the regulators further evidence to evaluate the safety and efficacy of the new medicine.

During this long process, money changes hands many times. A university filing for a patent creates a competing financial interest (CFI) for itself and for the researcher who made the discovery. Licensing a target or a lead to a company generates hefty revenue for the institution that owns the intellectual property. A pharmaceutical firm deciding on the best strategy to advance its drug-discovery program solicits the views of top scientists, who are financially rewarded for their advice. The pharmaceutical industry remunerates the clinicians in charge of running clinical trials. And, once the compound is ready for the patient, companies do not hesitate to use perks to turn doctors into strong advocates of the product-essentially extensions of their marketing machinery.

These CFIs can have a pernicious influence on translational research, the most negative effect of which is exposing patients to unsafe drugs. Our awareness of this harmful influence has increased over time, owing to highly publicized cases of dangerous drugs withdrawn from the market and to the advocacy of a series of journals, newspapers and scientists who have turned the fight against CFIs into a true crusade.

As a result of this increased awareness of CFIs, all parties involved have devised a series of measures to deal with them. Some institutions have banned their staff from consulting for or from owning stock. Policymakers have passed laws that prevent doctors from accepting presents from companies and companies from handing out gifts. Journals often ask authors to declare all of their CFIs as a prerequisite for publication of their work and may even ban some scientists from authoring reviews or editorials if they report a financial interest.

One thing that the items in this list have in common is their regard for CFIs as a monolithic scourge; what matters is that we expose all conflicts and show as little tolerance as possible for those who have them, regardless of their nature. But even though a lot has been written about this subject, it is seldom acknowledged that not all CFIs are equally insidious and that some are even necessary for the success of translational research.

Scientists who share their expertise with a company and clinicians who agree to conduct a clinical trial have to be compensated for their work in the same way that every other professional ought to be rewarded for his or her labor. To suggest that they should make their contributions freely is disingenuous, and to argue that they should not get involved at all can only slow the development of new medicines.

Academic institutions and their employees must be free to benefit financially from the fruits of the advances made in their laboratories, either by independently developing them into products or by selling them to the highest bidder. In the US, the Bayh-Dole Act has protected this right for nearly three decades, and CFIs that result from a university's right to enact this policy should not raise eyebrows.

The media have taken to heart their duty to report on cases in which CFIs affect the welfare of the public, and an increasing number of scientific journals rightly alert their readers to the CFIs of the authors of the articles they publish. However, a second issue that is seldom acknowledged is the profound inefficiency of the prevailing caveat emptor approach for ensuring the integrity of translational researchrecording the existence of CFIs and letting the readers make whatever they wish of that information.

There is evidence that published trials sponsored by a company with a financial interest in the outcome are more likely to work in favor of the sponsor. However, the fact remains that clinical trials are very expensive, and only the pharmaceutical sector has both the incentive and the deep pockets to pay for them. Furthermore, emphasizing evidence of this type shifts our attention away from a more serious concern: the possibility of scientific fraud.

If there is one reason we need to worry about CFIs, it is because they could lead to misconduct whenever a researcher has a financial incentive to fabricate data. So, if a clinical trial goes ahead on the basis of fraudulent preclinical work or if a drug is approved on the basis of false clinical data, the problem is more profound than can be addressed by the declaration of a CFI. Researchers or clinicians who produce sham data to continue receiving funds from a company must be subject to severe disciplinary actions from their institutions and funding bodies. Companies that conceal negative data from regulators to receive approval for their drug should face not only fines but also penalties as serious as criminal charges. In any case, the focus should move away from CFI declarations and toward exposing and punishing scientific misconduct.

A third issue that is rarely acknowledged is that the stridency with which the CFI crusaders fight financial interests has created a milieu in which pharmaceutical firms have increasingly less breathing space to conduct their business, leading to a further slowing of drug development. As a result, companies have begun to embark on their own crusade to change their image, looking for opportunities to collaborate with policymakers, journals and the public to promote transparency.

Although this initiative is to be welcomed, the irony is that some of the companies' ideas are so bold that the system does not seem to 
be ready for them. For example, in offering to help academic institutions keep track of the members of their staff who have dealings with the pharmaceutical industry, some companies are willing to publish lists of all the people who receive money from them, along with how much and for what concept. Here, it is the academic institutions that are dragging their feet, not being willing to deal with embarrassing disclosures that might arise or not even being able to manage this information.

There is no point in denying that pharmaceutical companies have a history of devious dealings that have rightfully tarnished their reputa- tion. But if they are now really serious about changing for the better, it would be a shame not to seize the opportunity for an armistice. We could start by abandoning the inquisitorial attitude with which CFIs are judged, trying instead to engage in a true dialogue among all stakeholders. It would also help if we stopped pretending that money is not one of the most powerful motivators to push innovations in healthcare. Let us instead accept that most financial interests do not represent a conflict as a matter of course and that the influence of money is negative only if it leads to scientific fraud-the one infidel we need to burn at the crusader's stake.

\title{
Autism and other developmental brain disorders
}

\author{
The Second Roche-Nature Medicine Translational Neuroscience Symposium on Autism and other developmental \\ brain disorders was a resounding success.
}

T en years ago, if someone had suggested that we organize a translational meeting on autism and related disorders, we would have regarded the idea as too premature. The fact that the Roche-Nature Medicine Translational Neuroscience Symposium on Autism and other developmental brain disorders, held on 17 and 18 April in Buonas, Switzerland, was one of the best meetings we have ever been involved in clearly illustrates how far this discipline has come in a decade.

The goal of the meeting was to explore how other neurodevelopmental diseases, particularly those with a monogenetic basis, could inform autism research. This goal was certainly met, but the meeting also highlighted the spectacular progress that has been made toward understanding and treating forms of mental retardation.

Successful translational research transforms molecular understanding of disease into a new therapy, a process that the accompanying figure (Fig. 1), which several speakers showed during their talks, depicts as a cycle. It was quite exhilarating to see how, for diseases such as neurofibromatosis type $\mathrm{I}$, tuberous sclerosis and fragile $\mathrm{X}$ syndrome, researchers are tantalizingly close to completing their first full turn of this circle.

For these diseases, investigators have a good genetic grasp, informative animal models, a working molecular understanding of pathology and 'druggable' targets. Clinically available compounds that can treat aspects of disease pathology in preclinical models are beginning to be tested in people. The challenge for researchers in this field is now twofold: to design rigorous clinical trials to investigate the true therapeutic effect of the existing molecules and to move away from the existing pharmacopoeia, instead developing the new generation of small molecules and other therapies that will make it to the clinic.

For some diseases, such as Rett syndrome, progress has also been solid, but is presently a couple of steps behind in the translational cycle. Although the animal models of Rett syndrome have been useful to the

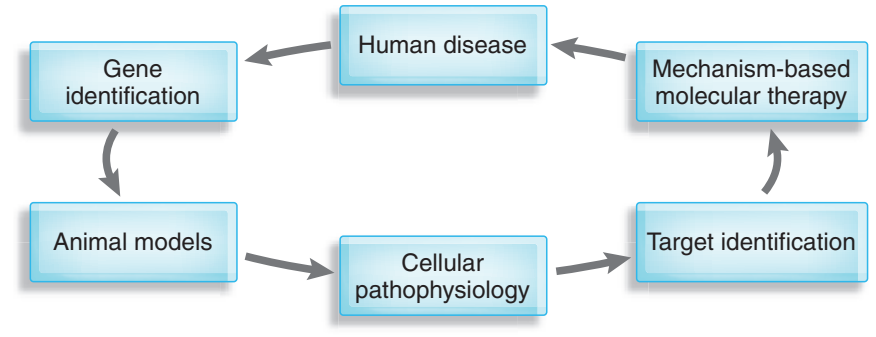

Figure 1 Translational research as a cycle that begins and ends with human disease.

point of showing that aspects of the disease might be reversible, we still lack mechanistic insight and specific molecular targets.

In the case of autism, spectacular progress has been made in understanding its genetic basis. Some of the alterations found in people with the disease, such as mutations in members of the shank and neuroligin protein families, have already been introduced into mice, with very intriguing results. But the fact remains that there is much work to do in creating animal models before investigators can figure out the molecular pathophysiology of autism. However, research into the other neurodevelopmental diseases is leaving a clearly marked trail for the autism field to follow as guidance.

We encourage you to visit the meeting's website (http://www.nature. com/natureconferences/tns2009/), where you can find a write-up of the event and, in due course, information about the next Translational Neuroscience Symposium. We are very proud of our partnership with Roche to create this symposium series, and we look forward to future, equally stimulating, meetings. 